\title{
MOBILE CLOUD COMPUTING APPLIED TO HEALTHCARE APPROACH
}

\author{
Omar AlSheikSalem ${ }^{1}$ and Muzhir Shaban Al-Ani ${ }^{2}$ \\ ${ }^{1}$ Department of Software Engineering, Applied Science University, Amman, Jordan \\ ${ }^{2}$ Department of Computer Science, College of Science and Technology, University of \\ Human Development, Sulaimani, Iraq
}

\begin{abstract}
In the past few years it was clear that mobile cloud computing was established via integrating both mobile computing and cloud computing to be add in both storage space and processing speed. Integrating healthcare applications and services is one of the vast data approaches that can be adapted to mobile cloud computing. This work proposes a framework of a global healthcare computing based combining both mobile computing and cloud computing. This approach leads to integrate all of the required services and overcoming the barriers through facilitating both privacy and security.
\end{abstract}

\section{KEYWORDS}

Mobile Computing, Cloud Computing, Medical Healthcare and Mobile Healthcare

\section{INTRODUCTION}

Internet was not just a research project that took place in 1969 but the project has become the unified language of the era and a dominant basis for most of the businesses around the world. In the past years, the Internet began to spread extremely fast across the world, therefore almost the whole world is covered with this online service; furthermore it has doubled in speed which was been taken into account as an advantage for improved ervices.

This issue increased the need for the emergence of smart mobile phones that have spread widely because of its excessive services and even became more influential when all of the internet services were available anytime anywhere $[1,2,3]$.

Recently, many technological terms exists such as utility computing distributed computing, cloud computing, mobile computing, grid computing, parallel computing, farm computing, and cluster computing etc. The mentioned terms are defined differently depending on the context and perspective depending on the changing technologies. Mobile and cloud computing are not a new terms, but goes along back to the beginning of the $2000[4,5]$. 
Smart phone devices and portable devices provides services that have been deployed in various fields of human lives including trading, commerce, smart home, healthcare, military, industry, education, public service etc. Currently, a smart phone device is not just a tool for communication, but it evolved to become an essential tool for many aspects in human lives [6, 7, 8].

\section{MOBILE, CLOUD AND HEALTHCARE COMPUTING}

Cloud computing is a new aspect of computing which change the understanding of mathematical computing based on personal computers or super computers to new aspect of computing that changes the concept of traditional computing (personal computers or super computers) to a cloud of computers or servers. In relation to cloud computing, the user has nothing to do with the service that he wants to deal with and how they work or the way they are constructed, but what the user is required to understand is to request the service and how to get to that type of service. Services provided by the cloud are not imited to the use of web applications only, but also it can be the tasks of IT management including the request of systems or a specific web appliance etc. $[9,10,11]$.

Mobile data communication has become a very important technology as users are allowed to transmit and receive data from point to point remotely anytime anywhere. This offers a main solution to the biggest problem of business people on the movement from one place to another. Mobile Computing concentrate more computing power in a small device. Mobile computing is a novel evolving technology that permits the transmission of images, data, animation, voice, and video through a mobile device or through any other device which is wireless enabled with no need to be connected to a fixed physical link, which enables users to do anything anytime and anywhere [12,13].

Years ago the industries are busy in manufacturing electronic devices and many of these devices are that concerned with medical electronic and digital devices and systems. Healthcare is the science that is related to the ability of improving human's health through the right diagnosis, then treatment, or prevention of illness, injuries, and diseases.. Healthcare Computing is a new aspect of using the advanced in computing to serve the Healthcare and patientcare services. The transition of traditional healthcare services are passed via many steps to be automated electronically. Healthcare computing including healthcare, patientcare, homecare, medical care, health information system, mobile computing, e-health, telemedicine and telehealth ... etc. [14, $15,16,17]$

\section{RELATED WORK}

Cloud and mobile computing are two new fields established in the last few years so most of the related works are published during these years and below some of these works.

Pragya Gupta and Sudha Gupta (2012) explained that smart phone and portable devices enable the user to have access to services in need anytime, anywhere. In addition they studied mobile cloud computing which integrates cloud computing with the environment of mobile technology 
and to overcome the barriers in relation to performance including bandwidth, storage space, battery consumption, and environment including, scalability, availability, heterogeneity and afterwards security including privacy and reliability [18].

Weiwen Zhang1, Yonggang Wen, and Dapeng Oliver Wu (2013), proposed the policy of scheduling for a collaborative execution on to mobile cloud computing. Regarding the execution, a mobile application is either executed on the mobile device itself or offloaded onto the cloud side. The goal of the design is to reduce the battery power energy consumed by mobile devices, to meet the time deadline if any [19].

C Shravanthi, H S Guruprasad, (2014) presented a questionnaire on the applications of mobile cloud computing applications, in addition to that the study presented the challenges, the current solutions and some ways to overcome such challenges facing mobile cloud computing. It was stated that mobile cloud computing is the integration of cloud computing into the environment of the mobile technology and the overcome of the challenges related to security, environment, and performance [20].

Zhou, Amir Vahid Dastjerdi, Rodrigo N. Calheiros, Satish Narayana Srirama, and Rajkumar Buyya, (2015) proposed an MCC offloading system prototype which takes into consideration various resources of the cloud including public clouds, mobile ad-hoc network, and cloudlet in order to offer an adaptive MCC service(s). The study proposed an algorithm of context-aware offloading decision intending of providing a code offloading decision(s) at runtime on to the process of selecting a wireless medium and of which prospective resources of the cloud, because the offloading location is based on the device's context [21].

Sheren A. El-Booz, Gamal Attiya and Nawal El-Fishawy (2016) This study proposed a system that improves the level of authentication level in security through the use of two techniques of authentication; time-based one-time password (TOTP) for the verification of the users of the cloud and the automatic blocker protocol (ABP) in order to protect the system from the intrusion of an unauthorized third party auditor. The study reveals an experimental results of how efficient and effective is the proposed system once shared data integrity is audited [22].

\section{HEALTHCARE MOBILE CLOUD COMPUTING (HMCC) APPROACH}

Healthcare services are created by the combination of all medical care services including doctors, hospitals, laboratories, pharmacies, and other support items. These services have been advancing step by step starting from traditional healthcare till cloud healthcare. Cloud computing and services are the future services that are not discovered till now as shown in figure (1). 


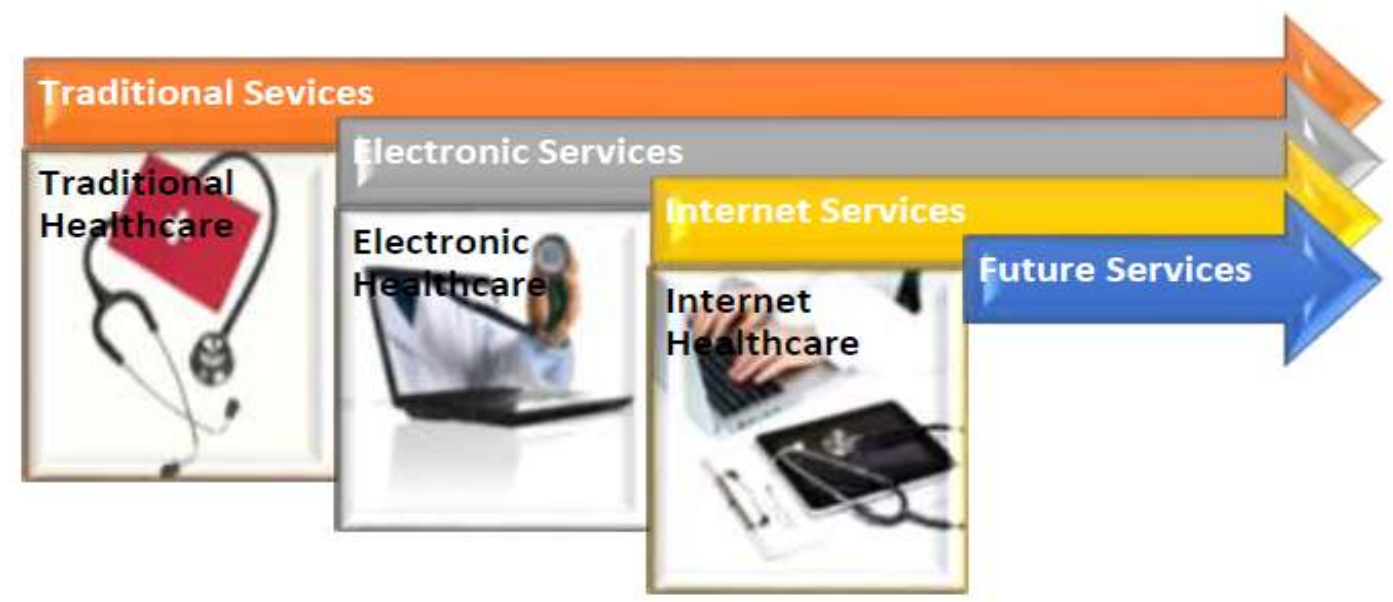

Figure (1) Developing of healthcare services

The integration of cloud and mobile computing lead to an extensive flexibility of accessibility of services. One of the major issues is integrating the healthcare services in addition to that the integration of mobile and cloud computing as presented in figure (2).

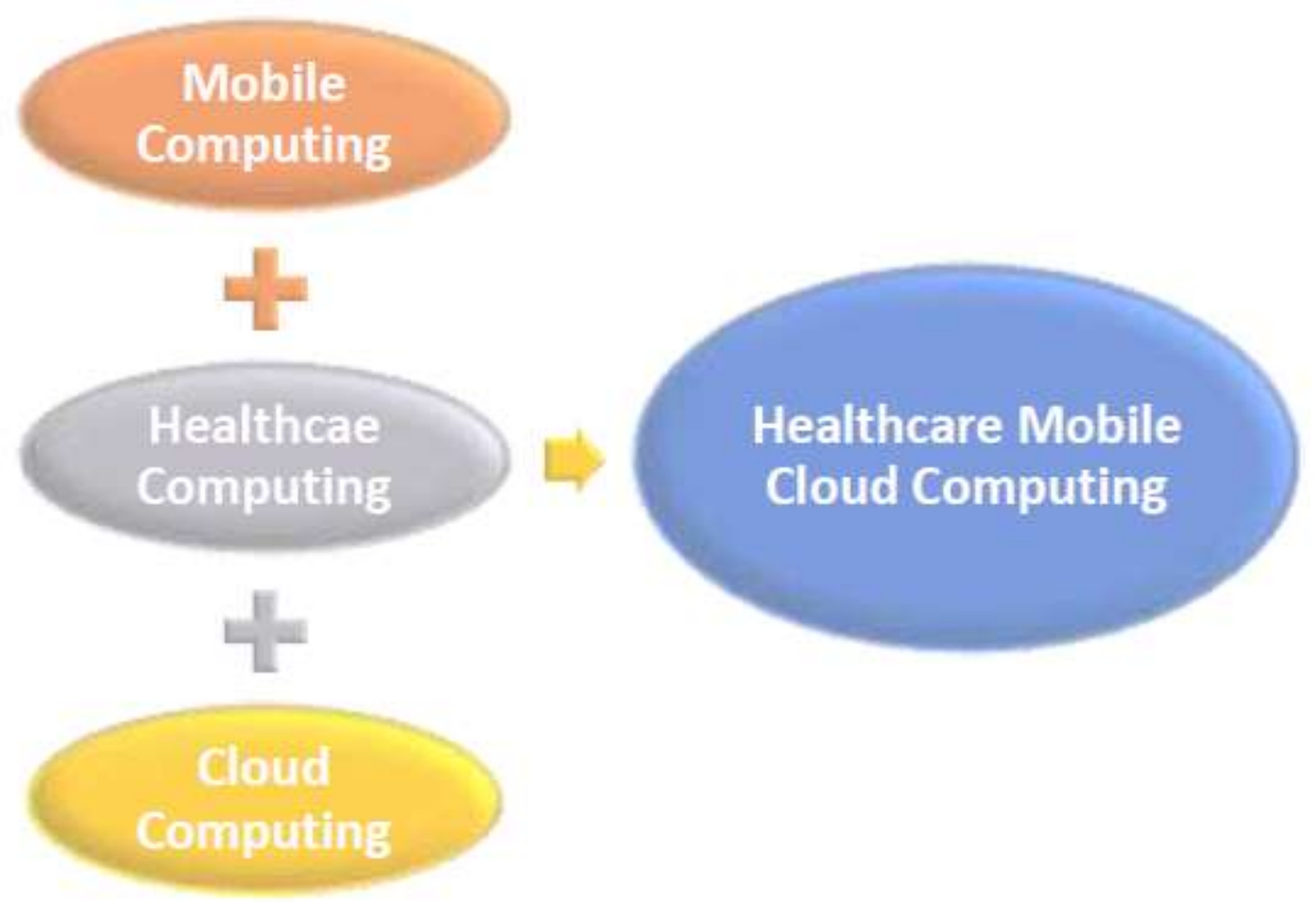

Figure (2) Integration of healthcare, mobile, and cloud computing 
The structure of healthcare, mobile, and cloud computing approach began by combining all wireless devices with the Internet via wireless service provider in which is connected to the cloud service provider that offers all requested applications as shown in figure (3). In this structure the user can access his/her healthcare demands applications via his/her mobile device(s) anywhere anytime then he/she can get his/her request using a web browser. The ability to process and store data is relocated from mobile devices to an influential and centralized computing platforms that are located in the cloud, in which the user is not concerned about the method and the technology he/she uses to get what he/she needs.

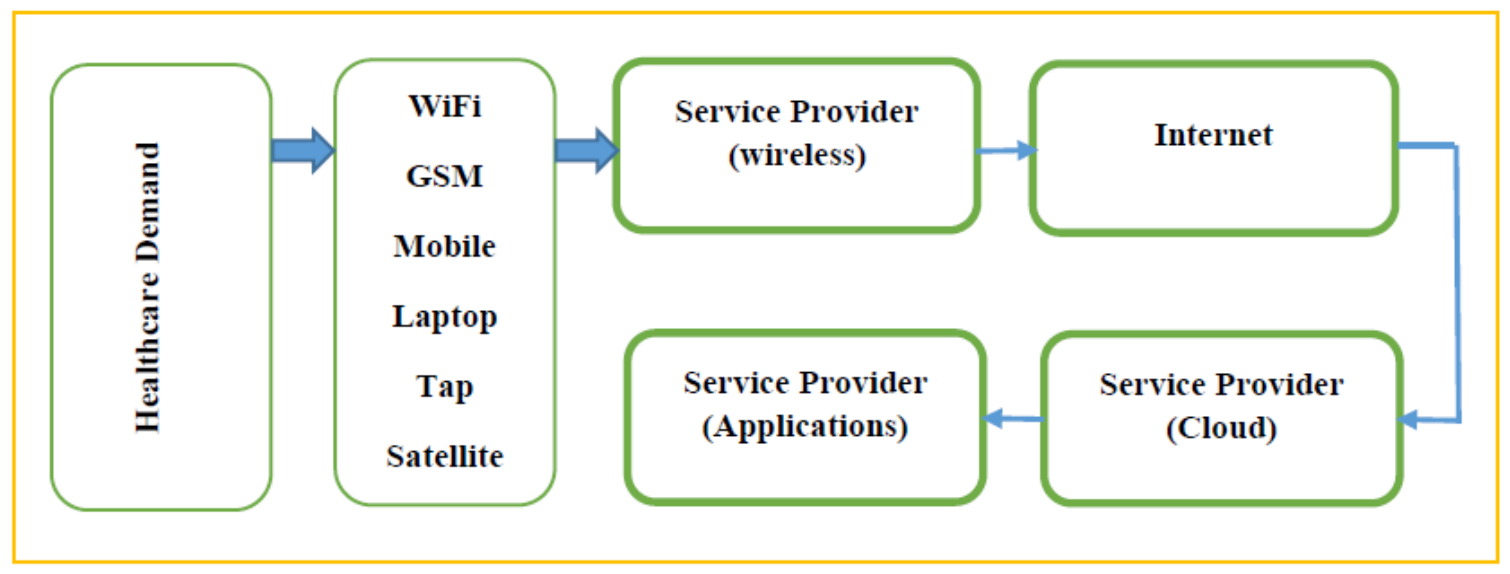

The structure of mobile cloud healthcare can be divided into three types of environment as shown in figure (4):

Cloud computing environment including infrastructure, platform, software and healthcare services; with their support of servers, databases, storage and systems.

Mobile computing environment including mobiles, satellite and Internet; with their hardware equipment and devices.

Mobile devices environment including advanced mobile technologies and devices. 


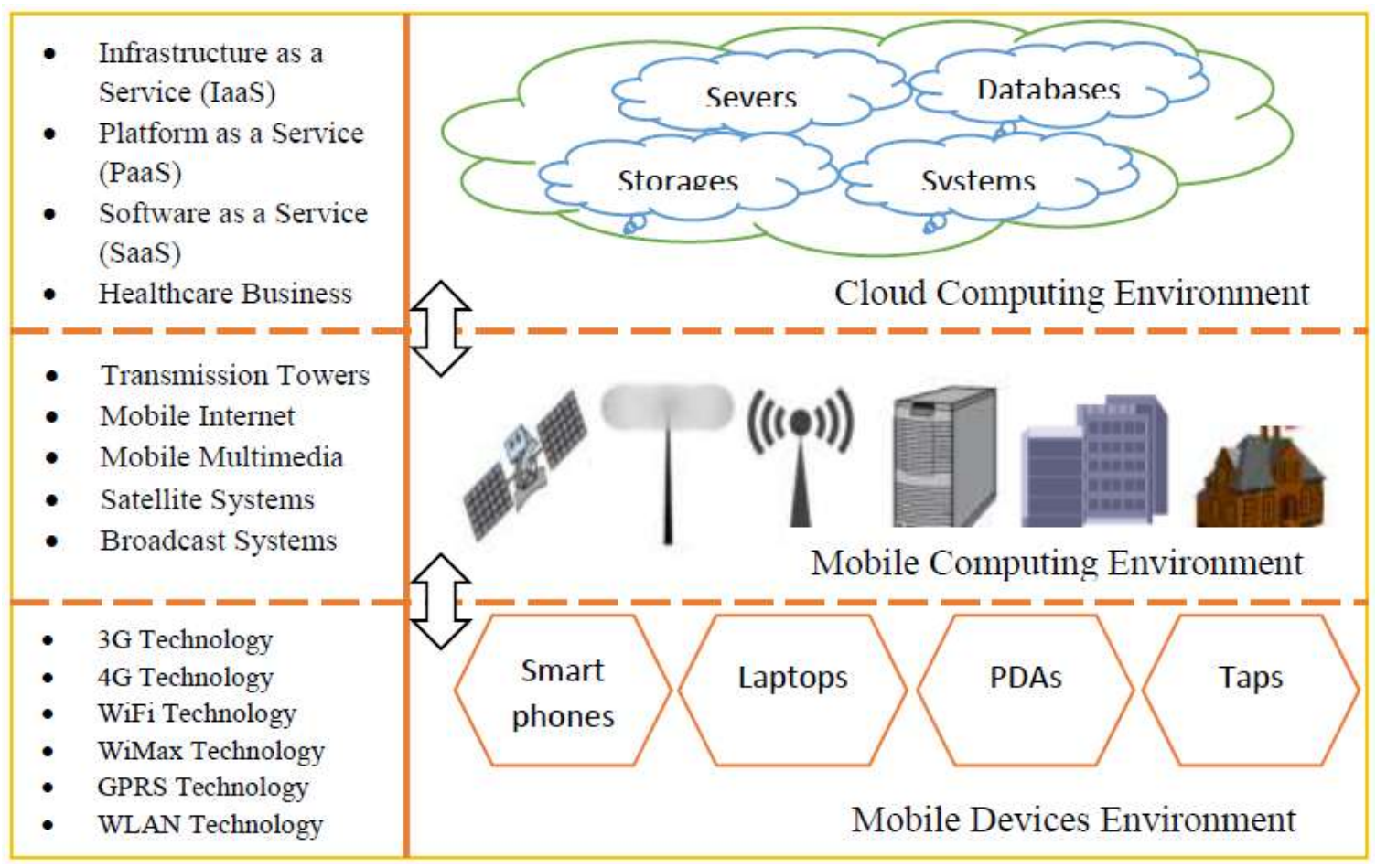

Figure (4) Mobile cloud healthcare structure.

\section{CONCLUSIONS}

Healthcare and patientcare are very important applications to be adapted via mobile cloud computing approach. The shifting from traditional healthcare model to consumer driven healthcare model is a very important aspect in this approach in which is moving forward to establish a direct private connection to the consumer patient model. This approach achieves a respectable performance of healthcare services anytime anywhere for both privacy and security of protecting confidential information of the consumer (patient). This issue opens a new future field of computing that lacks resources, including flexible architecture, adapted protocols, real time processing, huge storage, online services, privacy and security.

\section{ACKNOWLEDGEMENT}

The authors are grateful to the Applied Science Private University, Amman, Jordan, for the full financial support granted to this research. 
International Journal of Information Technology Convergence and Services (IJITCS) Vol.6, No.5, October 2016

\section{REFERENCES}

[1] R. Buyya, C. S. Yeo, S. Venugopal, J. Broberg, and I. Brandic, "Cloud computing and emerging IT platforms: Vision, hype, and reality for delivering computing as the 5th utility," Journal on Future Generation Computer Systems, vol. 25, no.6, Pages 599-616, 2009.

[2] R. Buyya, C. Yeo and S. Venugopal, "Market-oriented cloud computing: Vision, hype, and reality for delivering IT services as computing utilities," in10th IEEE International Conference on High Performance Computing and Communications, (HPCC '08), Pages 5-13, 2008.

[3] Muzhir Shaban Al-Ani, "The Road Map Revolution of Next Generation Mobile Commerce", International Journal of Business and ICT, Volume 1 Number 1-2, March-June 2015.

[4] Han Qi, Abdullah Gani, "Research on Mobile Cloud Computing: Review,Trend and Perspectives" in Proceedings of the Second International Conference on Digital Information and Communication Technology and its Applications (DICTAP), IEEE, Pages 195-202, 2012.

[5] Sean Marston, Zhi Li, Subhajyoti Bandyopadhyay, Juheng Zhang, Anand Ghalsasi, "Cloud Computing - The business perspective”, Decision Support Systems, Volume 51, Issue 1, Pages 176-189, 2011.

[6] B. Chun, S. Ihm, P. Maniatis, M. Naik, and A. Patti, "Clonecloud: Elastic execution between mobile device and cloud," in Proceedings of the sixth conference on Computer systems. ACM, Pages: 301314, 2011.

[7] Lei Yang, Jiannong Cao, Shaojie Tang, Tao Li, Alvin T.S. Chan, "A Framework for Partitioning and Execution of Data Stream Applications in Mobile Cloud Computing," in 5th International Conference on Cloud Computing (CLOUD), IEEE, Pages: 794-802, 2012.

[8] Muzhir Shaban Al- Ani \& Mohammed Salah Ibrahim, "Efficient Virtual Universities via Cloud Computing Environment", Journal of Emerging Trends in Computing and Information Sciences, Vol. 3, No.11 Nov, 2012.

[9] Hoang T. Dinh, Chonho Lee, Dusit Niyato and Ping Wang, A survey of mobile cloud computing: Architecture, applications, and approaches, wireless communications and mobile computing, Wireless Communications and Mobile Computing, 13 (2013), 1587- 1611.

[10] S. Abolfazli, Z. Sanaei, E. Ahmed, A. Gani and R. Buyya, Cloud-based augmentation for mobile devices: Motivation, taxonomies, and open issues, IEEE Communications Surveys and Tutorials 2014, 16 (2014), 337-368.

[11] Muzhir Shaban Al-Ani and Ghazi Ibrahem Raho, "Flying Virtual Cloud Computing Integrated System", (FVCIS) International Journal of Computer Trends and Technology (IJCTT), (IF=1.517), volume 30 Number 1 - December 2015

[12] L. F. Bittencourt, E. R. M. Madeira and N. L. S. D. Fonseca, Scheduling in hybrid clouds, IEEE Communications Magazine, 50 (2012), 42-47.

[13] L. F. Bittencourt, HCOC: A cost optimization algorithm for workflow scheduling in hybrid clouds, Journal of Internet Services \& Applications, 2 (2011), 207-227.

[14] U. Varshney, "Pervasive Healthcare", IEEE Computer Magazine vol. 36, no. 12, 2003, pp. 138-140

[15] Glickman SW, Baggett KA, Krubert CG, Peterson ED. Promoting quality: The healthcare organization from a management perspective. Int J Qual Health Care. 2007.

[16] Aghamollaei T, Zare SH, Bodat A. Patients perception and expectation about healthcare services in Bandarabas healthcare centres. Journal of Hormozgan University of Medical Sciences. 2007;11:1738.

[17] Muzhir Shaban Al-Ani, Raed I. Hamed \& Khattab M. Ali Alheeti, "The Mechanism of Monitoring and Tracking of Healthcare Systems", J. of university of Anbar for pure science : Vol.6:No.2 : 2012, Pages: 90-94.

[18] Pragya Gupta and Sudha Gupta, "Mobile Cloud Computing: The Future of Cloud", International Journal of Advanced Research in Electrical, Electronics and Instrumentation Engineering, Vol. 1, Issue 3, September 2012. 
International Journal of Information Technology Convergence and Services (IJITCS) Vol.6, No.5, October 2016

[19] Weiwen Zhang1, Yonggang Wen, and Dapeng Oliver Wu, "Energy-efficient Scheduling Policy for Collaborative Execution in Mobile Cloud Computing", 2013 Proceedings IEEE INFOCOM.

[20] C Shravanthi, H S Guruprasad, "Mobile Cloud Computing as Future for Mobile Applications", International Journal of Research in Engineering and Technology (IJRET), Volume: 03 Issue: 05, May-2014.

[21] Zhou, Amir Vahid Dastjerdi, Rodrigo N. Calheiros, Satish Narayana Srirama, and Rajkumar Buyya, "A Context Sensitive Offloading Scheme for Mobile Cloud Computing Service Bowen", 2015 IEEE 8th International Conference on Cloud Computing.

[22] Sheren A. El-Booz, Gamal Attiya and Nawal El-Fishawy, "A secure cloud storage system combining time-based one-time password and automatic blocker protocol", EURASIP Journal on Information Security (2016) 2016:13.

\section{AUTHORS}

Dr. Omar Al Sheik Salem is currently an assistant professor at Applied Science U niversity in Amman, Jordan. Dr. AlSheikSalem holds a Ph.D. degree in Computing from Bradford University, UK. His research interests are in Mobile TV, especially in the context of consumer needs and mobile advertisements, Ecommerce, multimedia, Mobile TV content, and new ways of advertising and interactive video.

Muzhir Shaban Al-Ani has received $\mathrm{Ph}$. D. in Computer \& Communication Engineering Technology, ETSII, Valladolid University, Spain, 1994. Assistant of Dean at Al-Anbar Technical Institute (1985). Head of Electrical Department at AlAnbar Technical Institute, Iraq (1985-1988), Head of Computer and SoftwareEngineering Department at Al-Mustansyria University, Iraq (1997-2001), Dean of Computer Science (CS) \& Information System (IS) faculty at University of Technology, Iraq (2001-2003). He joined in 15 September 2003 Electrical and Computer Engineering Department, College of Engineering, Applied Science

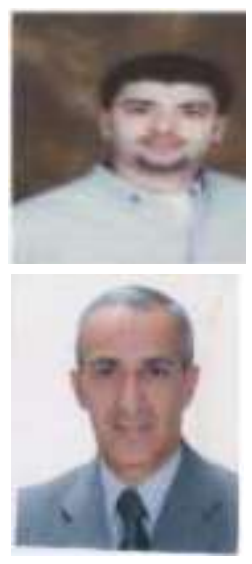

University, Amman, Jordan, as Associated Professor. He joined in 15 September 2005 Management Information System Department, Amman Arab University, Amman, Jordan, as Associated Professor, then he joined computer science department in 15 September 2008 at the same university. He joined in August 2009 College of Computer Science, Al-Anbar University, Al-Anbar, Iraq, as Professor. He joined in 2016 Department of Computer Science, College of Science and Technology, University of Human Development, Sulaimani, Iraq, as a Professor 